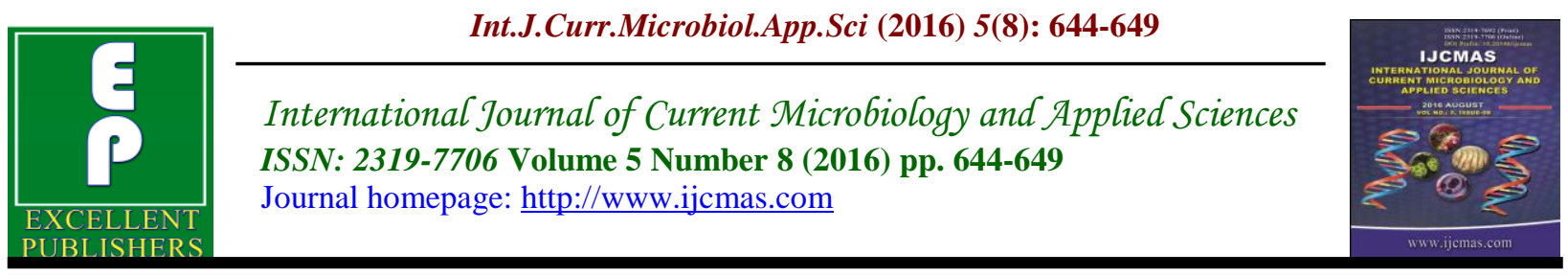

Original Research Article

http://dx.doi.org/10.20546/ijcmas.2016.508.072

\title{
A Study of Bacterial Culture Isolates and Antibiogram in Diabetic Foot Ulcer in GGG Hospital, Jamnagar, Gujarat, India
}

\author{
Neela C. Katara", Bindiya J. Ghedia and Mala Sinha \\ Department of Microbiology, Shree M.P. Shah \\ Government Medical College and GGG Hospital, Jamnagar, Gujarat, India \\ *Corresponding author
}

Keywords

Bacterial culture isolates, antibiogram, diabetic foot ulcer.

\section{Article Info}

Accepted:

23 July 2016

Available Online:

10 August 2016

\section{A B S T R A C T}

A diabetic foot is common complications of diabetes and cause of the hospitalization. Major increase in mortality among diabetic patients, observed over the past 20 years is considered to be due to the development of macro and micro vascular complications, including failure of the wound healing process. The individuals with diabetes have at least a 10 -fold greater risk of being hospitalized for soft tissue and bone infections of the foot than individuals without diabetes. This study was carried out to determine the bacteriological profile of infected diabetic foot ulcers and their antibiotic sensitivity pattern. Total 280 pus samples were collected from diabetic foot by using sterile swabs and they were processed. Study was carried out over the period of 5 months from January 2014 to may 2014 in microbiology department, Shri M.P. Shah Government medical College, GGGH, Jamnagar. A total of $77(27.5 \%)$ bacterial culture isolates were obtained from 280 samples with diabetic foot. Gram negative bacilli were more prevalent $(87.02 \%)$ than gram positive cocci (12.98\%). The antibiogram (antibiotic sensitivity pattern) of the bacteria were also studied. Gram negative bacilli were sensitive to Piperacillin+Tazobactum (79.10\%), Amikacin (40.29\%), Imipenem (38.80\%), Netilmicin (28.35\%) Gentamicin (17.91\%), Cefoperazone (14.92\%), Ceftazidim $(4.47 \%)$. Gram positive cocci were sensitive to Linezolid(100\%), Vancomycin (70\%), Clindamycin and Tetracycline (50\%), Azithromycin and Gentamicin (30\%), Cotrimoxazole (20\%), Cephalaxin(10\%). Knowledge on the antibiogram of the isolates will be helpful in determining the antibiotics for the treatment of diabetic foot ulcers rather than prescribing the empirical antibiotics.

\section{Introduction}

Diabetes mellitus is a metabolic disorder present with chronic hyperglycaemia and disturbances in carbohydrate, fat and protein metabolism. It is due to defects in insulin secretion, action or both. It manifested as damage or dysfunction in various organs like retina, kidney, Cardio vascular system,
Cerebro vascular system, bone, joints \& soft tissue like foot ulcer. It also includes physiological disturbances like sexual dysfunction. The major contributory factors for the high prevalence of the complications are; delayed diagnosis of diabetes, inadequate control of glycaemia, 
hypertension, and lack of awareness about the disease among majority of the public (Yashpal et al., 2012).

Diabetes mellitus is more prevalent within increasing age specially middle age and elderly people (Park et al., 2009).

Diabetic foot is infection, ulceration and/or gangrene of foot due diabetes mellitus. It is actually a triad of neuropathy, ischaemia and infection which required hospitalisation and prolonged stay. The presence of infection rapidly worsens the clinical picture often requiring limb amputation. Diabetic foot ulcers are common and estimated to affect $15 \%$ of all diabetics. In India, it is estimated that approximately 40,000 legs are being amputated every year, of which $75 \%$ are neuropathic feet which are potentially preventable (Yashpal et al., 2012, Bhavin Dalal et al., 2003). This study was carried out to determine the bacteriological profile in diabetic foot ulcers and their antibiotic sensitivity pattern.

\section{Materials and Methods}

\section{Study duration \& sample size}

Total 280 pus samples were studied over the period of 5 months from January 2014 to May 2014 in Microbiology department, M.P. Shah government medical College, GGGH, Jamnagar.

Pus samples were collected by using sterile swabs and they were processed. After obtaining proper patient informed consent, samples were collected from the deeper portion of the ulcers (ulcer base) by using 2 sterile swabs. The samples were collected by making a firm, rotatory movement with the swabs. One swab was used for Gram staining and the other was used for culture. A direct Gram stained smear of the specimen was examined. The specimens were inoculated onto blood agar, chocolate agar, Mac Conkey's agar. The inoculated plates were incubated at $37^{\circ} \mathrm{C}$ overnight and the plates were examined for growth on the next day. The further processing was done according to the nature of the isolate, as was determined by Gram staining, culture characteristic and their biochemical reactions.

The antibiogram (antibiotic sensitivity pattern) of the bacteria were also studied by the Modified Kirby Bauer disc diffusion method by using Mueller Hinton Agar according to CLSI (Clinical and Laboratory Standard Institute) guideline.

The antimicrobial discs which were used were those of Piperacillin/Tazobactum $(100+10 \mathrm{mcg})$, Amikacin(30mcg),Netilmicin( $30 \mathrm{mcg}$ ),Imipenam(10mcg),Ciprofloxacin(5 $\mathrm{mcg})$,Cefoperazone(30mcg), Gentamicin(10 mcg)for the Gram negative bacilli. Vancomycin (30 mcg), Linezoli (30 mcg), Azithromycin (30mcg), Clindemycin (2mcg), Tetracycline (30mcg), Cotrimoxazole $(25 \mathrm{mcg})$, Gentamicin (10mcg) were used to study the susceptibility patterns of the Gram positive cocci .

\section{Inclusion and exclusion criteria}

Total two swabs were collected in each \& every patients. One was tested by direct gram staining and results of direct gram staining were excluded from study while second was tested by culture method and results were included in study.

\section{Ethical clearance}

It was a retrospective analysis of samples collected for routine diagnosis, so ethical consideration was not necessary. 


\section{Results and Discussion}

A total of $77(27.5 \%)$ bacterial isolates were obtained from 280 pus samples with diabetic foot as shown in table 1 .

The study included $57(74.01 \%)$ males and $20(25.99 \%)$ females . The ages ranged from 20-80 years. Most numbers of foot infections were seen between the age group of 51-60 years followed by 41 to 50 years as shown in table 2.

Gram negative bacilli 67 (87.02\%) were more prevalent than gram positive cocci 10 $(12.98 \%)$ as shown in table 3.

A total of $67(87.02 \%)$ Gram negative bacilli were isolates among which Pseudomonas spp. $43(55.86 \%)$ was the commonest organism followed by Proteus spp. 8 (10.38\%), Klebsiella spp. $6 \quad(7.79 \%)$, Acinetobacter spp. 6(7.79\%) and E-coli 4(5.19\%). A total of 10(12.98\%) Gram positive cocci were isolates among which Staphylococus aureus 5 (6.5\%) was the commonest organism followed by Enterococcus spp 4 (5.19\%) and Coagulase Negative Staphylococcus aureus 01 $(1.29 \%)$.
In present study gram negative isolates were more sensitive to Piperacillin + Tazobactum $79.10 \%$ followed by Amikacin $40.29 \%$, Imipenem $38.80 \%$, Netilmicin $28.35 \%$ and Gentamicin $17.91 \%$ as shown in figure 1 .

In present study gram positive isolates were more sensitive to Linezolide 100\% followed by Vancomycin $70 \%$, Clindamycin and Tetracyclin 50\%, Azithromycin and Gentamicin $30 \%$, Cotrimoxazole $20 \%$ as shown in figure 2 .

In present study total of 77 bacterial isolates were obtained from 280 pus samples with diabetic foot as shown in table-1. The bacterial culture positivity rate is $27.5 \%$ in our study which is lower than the study of Jayshree et al 38\% (Jayshree Konar et al., 2013).

Rate of diabetic foot infections in male was $74.01 \%$ in current study as shown in table- 2 . Almost same result has been identified in study of Pathare et al., with $78.5 \%$ positivity rate in male (Mendes et al., 2012). This could because diabetes mellitus is more common in male and male are prone to trauma because of their outdoor occupation.

Table.1 Total positive cases.

\begin{tabular}{|l|l|l|}
\hline+ Ve (\%) & -Ve (\%) & Total (\%) \\
\hline $77(27.5 \%)$ & $203(72.5)$ & $280(100 \%)$ \\
\hline
\end{tabular}

Table.2 Age and sex distribution

\begin{tabular}{|l|l|l|l|}
\hline $\begin{array}{l}\text { Age } \\
\text { range }\end{array}$ & Male (\%) & Female (\%) & \% (n=77) \\
\hline $20-30$ & $03(3.89)$ & $01(1.3)$ & $04(5.19)$ \\
\hline $31-40$ & $03(3.89)$ & $02(2.60)$ & $05(6.49)$ \\
\hline $41-50$ & $15(19.49)$ & $06(7.80)$ & $21(27.29)$ \\
\hline $51-60$ & $29(37.66)$ & $08(10.39)$ & $37(48.05)$ \\
\hline $61-70$ & $04(5.19)$ & $02(2.60)$ & $06(7.79)$ \\
\hline $71-80$ & $03(3.89)$ & $01(1.3)$ & $04(5.19)$ \\
\hline Total & $\mathbf{5 7 ( 7 4 . 0 1 )}$ & $\mathbf{2 0}(\mathbf{2 5 . 9 9})$ & $\mathbf{7 7}(\mathbf{1 0 0})$ \\
\hline
\end{tabular}


Table.3 Isolated Gram negative and Gram positive organisms

\begin{tabular}{|c|c|}
\hline Organisms no $(\%)(n=77)$ & Total $(\%)$ \\
\hline Gram Negative Bacilli $67(87.02 \%)$ & \multirow{10}{*}{$77(100 \%)$} \\
\hline Pesudomonas spp $43(55.86 \%)$ & \\
\hline Proteus spp $08(10.38 \%)$ & \\
\hline Klebsiella spp $06(7.79 \%)$ & \\
\hline Acinetobacter spp $06(7.79 \%)$ & \\
\hline Escherichia coli $04(5.2 \%)$ & \\
\hline Gram Positive cocci $10(12.98 \%)$ & \\
\hline Staphylococcus aureus 05 (6.5\%) & \\
\hline Enterococcus 04 (5.19\%) & \\
\hline $\begin{array}{l}\text { Coagulase Negative Staphylococcus aureus } \\
01(1.29 \%)\end{array}$ & \\
\hline
\end{tabular}

Fig.1 Antibiotic sensitivity pattern of gram negative isolates

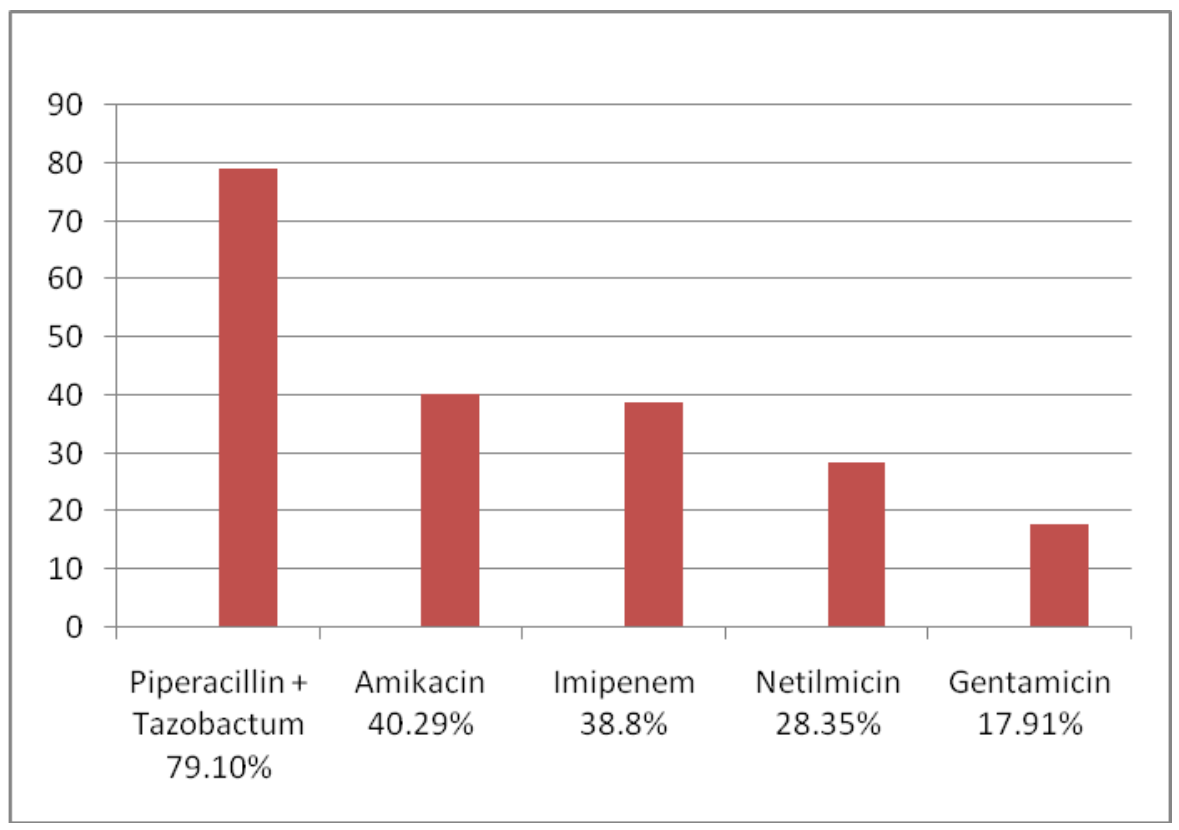


Fig.2 Antibiotic sensitivity pattern of gram positive isolates

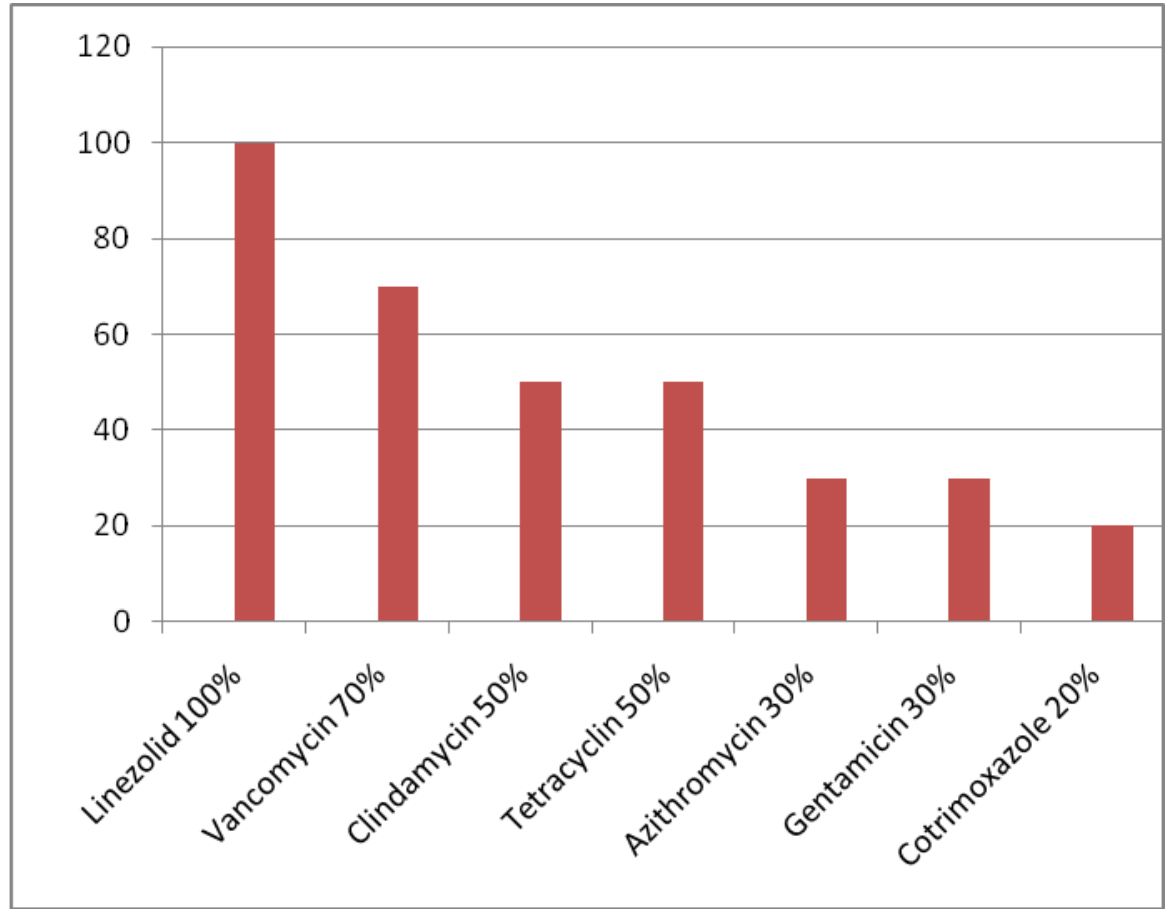

In present study diabetic foot infections were more common in 51 to 60 years $(37.66 \%)$ followed by 41 to 50 years $(19.49 \%)$ as shown in table-2. The most common age group in study of Leela et al., was 51 to 60 years $(45.3 \%)$ followed by 41 to 50 years $(31.33 \%)$ (Leela Rani et al., 2013) which is little higher than current study. Both the study shows that diabetic foot infection cases are increasing with advancing age, exact reasons for this are unknown. but may be due to increasing diabetes with advancing age which favour the bacterial infection.

In present study GNB $(87.02 \%)$ were more common than GPC $(12.98 \%)$ as shown in table-3. High rate by GNB than GPC was also noticed by Gadepalli $R$ et al with $51.4 \%$ participation by GNB \& $33.3 \%$ participation by GPC (Gadepalli et al., 2006). Most common bacteria among the GNB category was Pseudomonas spp $(55.86 \%)$ while from GPC category it was Staphylococcus aureus $(6.5 \%)$ in our study as shown in table-3. Some research also suggest highest infection rate by Pseudomonas spp (30.67\%) from GNB category and Staphylococcus aureus (17\%) from GPC category in diabetic foot ulcer (Jain Manisha et al., 2012; Vaidehi Mehta et al., 2014).

Sensitivity pattern of present study suggest that all GNB isolates from diabetic foot ulcer were most sensitive to Piperacillin + Tazobactum $(79.10 \%)$ and less sensitive to other tested drugs as shown in figure-1, while GPC from diabetic foot ulcer were most sensitive to Linezolide (100\%) and less sensitive to other tested drugs as shown in figure-2. Research by Vaidehi et al indicates that GNB isolates from diabetic foot ulcer were also most sensitive to Piperacillin + Tazobactum but with sensitivity rate is almost more than half about 38\% while GPC isolates shows same result with $100 \%$ sensitivity rate to Linezolide.

In conclusion, in present study, Gram negative bacilli were more predominant than 
Gram positive cocci. Pseudomonas species and staphylococcus aureus were responsible for most of the Diabetic foot infections .Gram negative bacilli were most sensitive to Piperacillin+Tazobactum, and Gram positive cocci were most sensitive to Linezolid. Knowledge on the antibiogram of the isolates will be helpful in determining the antibiotics for the treatment of diabetic foot ulcers rather than prescribing the empirical antibiotics.

\section{References}

Bhavin Dalal, Parag Dalal. 2003. Handbook of medicine and surgery. $1^{\text {st }}$ edition. Puls Publishers.

Gadepalli, R., Dhawan, B., Sreenivas, V., Kapil, A., Ammini, A.C., Chaudhry, R. 2006. A Clinico-microbiological Study of Diabetic Foot Ulcers in an Indian Tertiary Care Hospital. Diabetes Care, 29:1727-32.

Jayashree Konar, Sanjeev Das. 2013. Bacteriological profile of diabetic foot ulcers, with a special reference to antibiogram in a tertiary care hospital in eastern India. J. Evol. Med. Dent. Sci., Vol. 2, Issue 48, December 02; Page: 9323-9328.

Mendesa, J.J., Marquescosta, C., Viela., J., Neves, N., Candeias, P., Cavaco-Silva,
J., Melo cristino. 2012. Clinical and bacteriological survey of Diabetic foot infections in Lisbon. Diabetes Res. Clin. Practice, 95:153-161.

Jain Manisha, Patel Mitesh, H., Sood Nidhi, K., Modi Dhara, J., Vegad, M.M. 2012. Spectrum of microbial flora in diabetic foot ulcer and its antibiotic sensitivity pattern in tertiary care hospital in Ahmedabad, Gujarat, 2(3): 354.

Park, K. 2009. Textbook of preventive and social medicine. $20^{\text {th }}$ edition.M/s Banarasidas. Bhanot Publishers.

Leela Rani. 2013. Bacteriological profile of Diabetic Foot infection. IJPRBS, 2(2): 36-45.

Sivaraman Umadevi, Shailesh Kumar, Noyal Mariya Joseph. 2011. Microbiological study of diabetic foot infections. Indian J. Med. Specialities, 2(1): 12-1.

Vaidehi, J., Mehta, Kunjan, M., Kikani, Sanjay, J., Mehta. 2014. Microbiological profile of diabetic foot ulcers and its antibiotic susceptibility pattern in a teaching hospital, Gujarat. Int. J. Basic \& Clin. Pharmacol., 3(1): 93.

Yashpal munjal. 2012. API textbook of Medicine. $9^{\text {th }}$ edition. Jaypee Brothers Medical Publishers.

\section{How to cite this article:}

Neela C. Katara, Bindiya J. Ghedia and Mala Sinha. 2016. A Study of Bacterial Culture Isolates and Antibiogram in Diabetic Foot Ulcer in GGG Hospital, Jamnagar, Gujarat, India. Int.J.Curr.Microbiol.App.Sci. 5(8): 644-649. doi: http://dx.doi.org/10.20546/ijcmas.2016.508.072 